\title{
Clonal Dynamics of Nasal Staphylococcus aureus and Staphylococcus pseudintermedius in Dog-Owning Household Members. Detection of MSSA ST ${ }^{398}$
}

\author{
Elena Gómez-Sanz, Carmen Torres, Sara Ceballos, Carmen Lozano, Myriam Zarazaga*
}

Área Bioquímica y Biología Molecular, Universidad de La Rioja, Logroño, Spain

\begin{abstract}
The objective of this study was to investigate the dynamics of nasal carriage by Staphylococcus aureus (SA) and Staphylococcus pseudintermedius (SP) among healthy dog-owning household members involved in 7 previous index cases of suspected anthropozoonotic $(n=4)$ and zoonotic $(n=3)$ interspecies transmission [4 direct cases, identical SA $(n=3)$ or SP $(n=1)$ in owner and dog; three indirect, SP in owner $(n=2)$ or SA in dog $(n=1)]$. Co-carriage with methicillin-resistant coagulase-negative staphylococci (MRCoNS) was also evaluated. Sixteen owners and 10 dogs were sampled once every three months for one year. In total, 50 SA and 31 SP were analysed by MLST, and SA also by spa typing. All isolates were subjected to Apal/Smal-PFGE and antimicrobial resistance and virulence profiles were determined. All index owners were persistent SA carriers in all direct-anthropozoonotic transmission cases, while only one dog was persistent SA carrier. Owner and dog exhibited a persistent SP carriage status in the direct-zoonotic transmission case. SP was maintained in the index human over time in one indirect-zoonotic transmission case. Only one SP was methicillin-resistant. SA belonged to genetic backgrounds of MRSA pandemic clones: CC45, CC121, CC30, CC5 and CC398. Three individuals carried a MSSA t1451-ST398 clone with the $\mathrm{erm}(\mathrm{T})-\mathrm{cadD} / \mathrm{cadX}$ resistance genes. SA or SP were persistently detected in the nasal cavity of $7(43.8 \%)$ and 2 (12.5\%) owners, and in one and 2 dogs, respectively. SA was recovered as the single species in 10 owners and in one dog; SP in 3 owners and 4 dogs; and both bacterial species in one owner and 4 dogs. Co-carriage of SA or SP with MRCoNS isolates was common (30.7\%). This is the first study on the dynamics of nasal carriage of SA and SP in healthy pet-owning household members. Dog-contact may play a role in the staphylococcal species distribution of in-contact individuals.
\end{abstract}

Citation: Gómez-Sanz E, Torres C, Ceballos S, Lozano C, Zarazaga M (2013) Clonal Dynamics of Nasal Staphylococcus aureus and Staphylococcus pseudintermedius in Dog-Owning Household Members. Detection of MSSA ST ${ }^{398}$. PLoS ONE 8(7): e69337. doi:10.1371/journal.pone.0069337

Editor: Holger Rohde, Universitätsklinikum Hamburg-Eppendorf, Germany

Received February 21, 2013; Accepted June 8, 2013; Published July 9, 2013

Copyright: (C) 2013 Gómez-Sanz et al. This is an open-access article distributed under the terms of the Creative Commons Attribution License, which permits unrestricted use, distribution, and reproduction in any medium, provided the original author and source are credited.

Funding: This study was financially supported by Projects SAF2009-08570 and SAF2012-35474 from the Ministerio de Economía y Competitividad of Spain. EGS has a fellowship from the Gobierno de La Rioja of Spain, and CL has a fellowship from the Ministerio de Economía y Competitividad of Spain. The funders had no role in study design, data collection and analysis, decision to publish, or preparation of the manuscript.

Competing Interests: The authors have declared that no competing interests exist.

*E-mail: myriam.zarazaga@unirioja.es

\section{Introduction}

Staphylococcus aureus (SA) and Staphylococcus pseudintermedius (SP) form part of the normal microbiota of the nares, skin and mucous of humans and dogs, but they are also opportunistic pathogens $[1,2]$. SA can be also found in dogs at moderate rates $(<20 \%)$ with a suggested anthropozoonotic origin, although the direction of transmission has not yet been elucidated [3-7]. Humans can carry SP at very low frequencies [8,9], while higher rates are generally detected among individuals with regular dog contact $(\leq 4.5 \%)$ $[2,3,7,10,11]$.

Analysis of the colonization dynamics over time is essential to address the real carriage status of a positive individual and for a better understanding of the interaction among staphylococci in the distinct hosts. However, very limited data do exist on the longitudinal carriage of SP in humans in contact to dogs [1214], and none among healthy individuals. A couple of recent studies on the carriage dynamics of methicillin-resistant SP (MRSP) in dogs and in-contact humans reported humans to be rarely colonized by MRSP for prolonged periods of time [13,14]. Various reports have focused on the SA colonization dynamics in humans, including specific populations, such as homeless people, drug users, healthy infants, health care workers, subjects with staphylococcal (previous) infection [15-22] or, more recently, in individuals in contact to livestock $[23,24]$. Nonetheless, to our knowledge, no longitudinal studies on SA from pet-owning humans and their pets have been reported to date.

On the other hand, humans can be carriers of methicillinresistant coagulase negative staphylococci (MRCoNS), which normally harbor multiple antimicrobial resistance genes [25-27]. Although these bacteria are considered reservoirs for the exchange of genetic material between different staphylococcal species [2831], co-carriage of SA or SP and MRCoNS in humans and incontact pets has not been previously analyzed.

In this study, we investigated the dynamics of SA and SP nasal carriage of dog-owning household members with previous suspected cases of SA or SP interspecies transmission [7] to evaluate the individual carriage status and to estimate the direction of bacterial transmission. In addition, we sought to assess the cocarriage of these bacterial species and MRGoNS, to appraise the possible risk of gene transfer between those microorganisms. Our results suggest that pet-owning may play a relevant role in the staphylococcal species distribution of in-contact individuals. 


\section{Materials and Methods}

Description of Investigated Households and Index Cases

Healthy owners and dogs coming from 7 households with previous description of suspected SA (4 cases, 1-4) or SP (3 cases, 5-7) interspecies transmission, recently detected in a study performed at the University of La Rioja (Spain) [7], were further investigated in the present study. Index cases were defined as cases in which SA or SP interspecies transmission is presumed to have occurred in original sampling (named T0), between at least one owner and one pet. All individuals tested were healthy and none has suffered from previous SP or SA infection. Direct interspecies transmission was considered when both, owner and dog, exhibited an identical SA or SP clone. Indirect interspecies transmission was defined as the presence of SA in dog but not in human, or SP in owner but not in dog. Figure 1 shows the composition of the residences, the bacterial species responsible and the different types of suspected interspecies transmission.

In the former study, household number assigned to the different index cases was as follows (case number in the present study): household no.1 (case 1), household 4 (case 2), household 2 (case 3), household 23 (case 4), household 5 (case 5), household 12 (case 6) and household 11 (case 7) [7]. Clone nomenclature used in this study is indicated as bacterial species and methicillin resistance pattern+Multilocus Sequence Type (MLST)+Pulsed-Field-GelElectrophoresis (PFGE) pattern. Index clones, defined as clones detected in index cases are shown in Figure 1 [7]. Several further isolates, not involved in the interspecies transmission index cases, were detected in $\mathrm{T} 0$ in the coexisting individuals (Figure 1). In total, 17 isolates (12 SA and $5 \mathrm{SP}$ ) obtained in T0 from the 7 households were included in the current study.

None of individuals tested presented occupational exposure to pets or the healthcare system. Both owners from cases 1, 3 and 6 were couples (range of age 27-30) while those from cases 2 and 7 were mother and/or grandmother (50-75 years old) plus 2 or 3 adults (son, daughter) (22-28). Owners from cases 4 and 5 (aged
27-35) lived alone with their dog. Individuals from cases 6 and 7 lived in rural areas.

\section{Study Design}

In addition to 7 owners and 5 dogs involved in T0 index cases (named with the letter "a" and gray shaded in Figure 1), all coexisting individuals were also investigated (9 owners and 5 dogs) (Figure 1). Nasal samples from the anterior nares of 16 owners and 10 dogs were studied once every 3 months for one year (5 sampling times, T0 to T4) with a total of 130 samples analyzed. Sixty-four samples were recovered from owners and 40 samples from dogs during T1-T4 and completely characterized in this study. In addition, 26 samples from T0 from the former study were considered. Nasal co-carriage of SA and/or SP with MRCoNS was also investigated. All persons completed a written informed consent and agreed that their dog/s were also included. Sampling was approved by the Medical Ethical Committee of La Rioja (Permit Number: METC 09-399/C). Sterile swabs were provided to the owners in addition to detailed description of the sampling procedures. The owners swabbed themselves and the nares of their dogs. Nasal swabs were collected from the household owner within 24-48 $\mathrm{h}$ after sampling and they were immediately processed or eventually stored at $-20^{\circ} \mathrm{C}$ until further analysis.

\section{Definition of the Carriage Status and Interspecies Transmission Dynamics}

Subjects positive for SA or SP in at least four of the five samplings (including T0) were considered persistent carriers; those positive in two or three samplings were defined as intermittent carriers; individuals positive in a single sampling were reported sporadic carriers; and those negative throughout the study were defined as non carriers. It is important to remark the possibility that individuals who carry both bacterial species exhibited different carriage status per bacterial species. Dynamics of the interspecies transmission cases over time was defined likewise (persistent, intermittent, and sporadic).
- Index SA clone
- Different SA clones within the same household
- Index SP clone
$\Delta \quad$ Distinct SP clones within the same household
$\square \quad$ Different concurrent MRCoNS clones within the same household
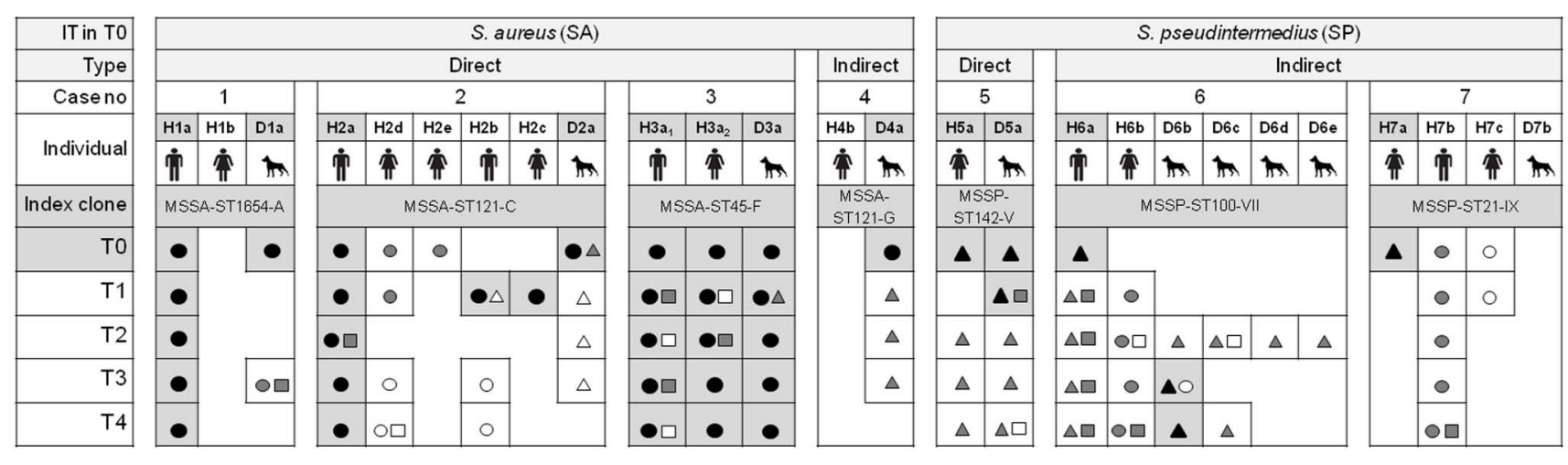

Figure 1. Schematic representation of the dynamics of carriage of the seven households investigated. MRCoNS, methicillin-resistant coagulase negative staphylococci. IT, bacterial species responsible for interspecies transmission. Type, type of interspecies transmission (Direct, Indirect). Case no, number of the households investigated in this study. Individuals are named H (for human) or D (dog) followed by the case number ( 1 to 7$)$ and a lower-case letter to differentiate subjects per household; if necessary, a lower script number was added. Nomenclature of individuals involved in index cases (T0) is displayed with gray background. T0 to T4, the different samplings. Index clone, S. aureus or S. pseudintermedius clones responsible for interspecies transmission in index cases (T0).

doi:10.1371/journal.pone.0069337.g001 


\section{Isolation and Identification of SA, SP and MRCoNS Isolates}

Samples were inoculated in Brain-Heart-Infusion broth (BHI, Difco) supplemented with $6.5 \% \mathrm{NaCl}$ and incubated at $37^{\circ} \mathrm{C}$ for $24 \mathrm{~h}$. One-hundred microliters were seeded on Oxacillin-Resistant-Staphylococcal-Agar-Base (ORSAB; OXOID) plates supplemented with $2 \mathrm{mg} / \mathrm{L}$ of oxacillin for the isolation of MR staphylococcal isolates, either SA, SP or CoNS. Seventy microliters were inoculated in parallel on Manitol-Salt-Agar (MSA; BD) plates for the isolation of SA and SP. Plates were incubated at $35^{\circ} \mathrm{C}$ for $24-48 \mathrm{~h}$. All colonies with different morphology were subcultured on BHI agar (Difco) and further studied. Preliminary identification of isolates was based on colony morphology, Gram staining, and catalase and DNase activities. A species-specific multiplex PCR was performed to identify SA and S. intermedius/SP isolates [32]. Discrimination between $S$. intermedius and SP was conducted by restriction fragment length polymorphism of the pta gene [33]. The presence of mecA gene was investigated by PCR in all isolates [34]. Identification of MRCoNS was performed by amplification and sequencing of the $\operatorname{sod} A$ gene in all mecA positive CoNS isolates [35]. Only one MRCoNS isolate per individual was further characterized when isolates belonged to the same bacterial species.

\section{Analysis of the Clonal Relatedness of Isolates}

The genetic relatedness of all isolates obtained (SA, SP and MRCoNS) was investigated by PFGE of total DNA restricted with SmaI or ApaI macrorestriction enzymes as previously described [36]. For SA and MRCoNS isolates, PFGE running conditions were those recommended by the HARMONY protocol [36]. SA isolates non-typeable with $S m a \mathrm{I}$ were subjected to ApaI-PFGE and run for $20 \mathrm{~h}$ at $6 \mathrm{~V} / \mathrm{cm}$ using pulsed time ramping from 2 to $5 \mathrm{~s}$ [37]. SmaI-digested plugs obtained from SP isolates were run for $24 \mathrm{~h}$ at $5.6 \mathrm{~V} / \mathrm{cm}$ using pulse times from 2 to $5 \mathrm{~s}$ [38]. Isolates were considered a unique clone when they showed up to three bands difference in PFGE band patterns and subclones when PFGE band patterns differed between 1-3 bands [39]. The different SA patterns were distinguished by capital letters, major SP patterns by Roman numbers and MRCoNS by Arabic numbers. Subclones were indicated with the major lettering type followed by a lower case letter.

\section{Molecular Typing of SA and SP Isolates}

All SA isolates were subjected to spa typing as previously described [40] and sequences were analyzed using Ridom StaphType software version 2.0.21 (Ridom GmbH). The last recovered isolate of each novel clone per household was selected as representative strain for in-depth molecular characterization. MLST was performed as recommended (http://www.mlst.net) on representative SA isolate. MLST was determined in all SP isolates; for this, five housekeeping genes were amplified and sequenced (pta, cpn60, tuf, 16S rRNA and agrD) followed by assignment of alleles by comparison to those present in the NCBI nucleotide database and using a key table for MLST typing of $S$. intermedius group (SIG) isolates [38].

\section{Antimicrobial Resistance Profile of Isolates}

Susceptibility testing to 17 antimicrobial agents was performed in all isolates obtained by agar disk-diffusion method [41]. Antimicrobials tested were as follows ( $\mu \mathrm{g} /$ disk): penicillin $(10 \mathrm{U})$, oxacillin (1), cefoxitin (30) erythromycin (15), clindamycin (2), gentamicin (10), kanamycin (30), streptomycin (10U), tobramycin (10), tetracycline (30), trimethoprim-sulfamethoxazole
$(1.25+23.75)$, chloramphenicol (30), ciprofloxacin (5), mupirocin (200), fusidic acid (10), vancomycin (30), and linezolid (30). Methods and breakpoints followed for streptomycin and fusidic acid were those recommended by the Société Française de Microbiologie (http://www.sfm-microbiologie.org). Inducible or constitutive clindamycin resistance was determined by the doubledisk diffusion test (D-test) [41].

Presence of 32 antimicrobial resistance genes was investigated by $\mathrm{PCR}$ in all representative $\mathrm{SA}$ and $\mathrm{SP}$ strains and in all MRCoNS isolates [34,42]. Antimicrobial resistance genes tested were as follows: $\operatorname{mec} \mathrm{A}, \operatorname{bla} Z, \operatorname{tet}(\mathrm{K}), \operatorname{tet}(\mathbf{M}), \operatorname{tet}(\mathrm{L}), \operatorname{tet}(\mathrm{O}), \operatorname{erm}(\mathrm{A}), \operatorname{erm}(\mathrm{B})$, $\operatorname{erm}(\mathrm{C}), \operatorname{erm}(\mathrm{T}), m p h(\mathrm{C}), m s r(\mathrm{~A}), m s r(\mathrm{~B}), \operatorname{aacA}-a p h D, \operatorname{aphA3}, \operatorname{aadE}, \operatorname{aadD}$, aadA, str, sat4, dfr( $(\mathbf{A}), d f r(\mathrm{D}), d f r(\mathrm{G}), d f r K, c_{p} t_{p 221}, c_{p} t_{p C 194}, c_{p} t_{p C 223}$, fexA, $c f r$, mupA, fusB and fus $C$.

\section{Detection of Virulence Genes}

Presence of the Panton Valentine leukocidin (PVL) genes (lukS/ $F-P V$ was determined in all isolates by PCR [7]. The leukocidins $l u k E / D$ and $l u k M$, exfoliatins $e t a$ and $e t b$, the toxic-shock syndrome toxin gene $t s t$ and 18 enterotoxin genes were likewise investigated in all representative SA strains [7]. These isolates were additionally tested by PGR for the presence of the human-specific immune evasion cluster (IEC) genes encoding the chemotaxis inhibitory protein $(c h p)$, staphylococcal complement inhibitor $(s c n)$ and staphylokinase $(s a k)$, enclosed within prophage $\phi 3$ [43].

The presence of leukocidin $l u k S / F-I$, exfoliatins siet, exp $A$ and $\exp B, 18$ enterotoxin genes and the species specific sec canine and se-int genes were also investigated by PCR in all representative SP strains [7].

\section{Results}

\section{SA Isolates and Clones Recovered and SA Population Structure}

All isolates recovered from the 7 households are schematically represented in Figure 1.

Thirty-two SA isolates were obtained from 16 owners and 6 isolates were recovered from 10 dogs. The $12 \mathrm{SA}$ isolates obtained in $\mathrm{T} 0$ were also considered [7]. In total, $50 \mathrm{SA}$ isolates were recovered from the 7 cases.

Based on the PFGE band profile of the 50 SA isolates, 10 SA clones were detected (Table 1). Ten different spa types (Table 2) and 7 STs (ST1654, ST121, ST5, ST398, ST45, ST30, and a new ST registered as ST2619) were observed. Clone MSSA-ST45-F in owner $\mathrm{H}_{3} \mathrm{a}_{1}$, exhibited 3 different but related spa types along the sample year $(\mathrm{T} 0 \rightarrow \mathrm{t} 073 ; \mathrm{T} 1 \rightarrow \mathrm{t} 116 ; \mathrm{T} 2 \rightarrow \mathrm{t} 026 ; \mathrm{T} 3-4 \rightarrow \mathrm{t} 073)$ (Table 2).

\section{SP Isolates and Clones Recovered and SP Population Structure}

Eight SP isolates were recovered from 16 owners and 18 isolates from 10 dogs. Including the $5 \mathrm{SP}$ isolates obtained in the former report (T0) [7], a total of $31 \mathrm{SP}$ isolates were recovered from the 7 cases.

Based on their PFGE band profile, 9 SP clones and 9 distinct STs (ST71, ST77, ST6, ST42, ST142, ST185, ST100, ST70 and ST21) were identified (Table 1). Four subclones (VIIIa to VIIId) of MSSP-ST70-VIII were detected (Table 1).

\section{Antimicrobial Resistance Profile of SA and SP Isolates}

All SA isolates were susceptible to oxacillin and cefoxitin and were $m e c \mathrm{~A}$ negative (Table 1). All SA isolates from the same clone presented identical antimicrobial resistance phenotypes. None of 


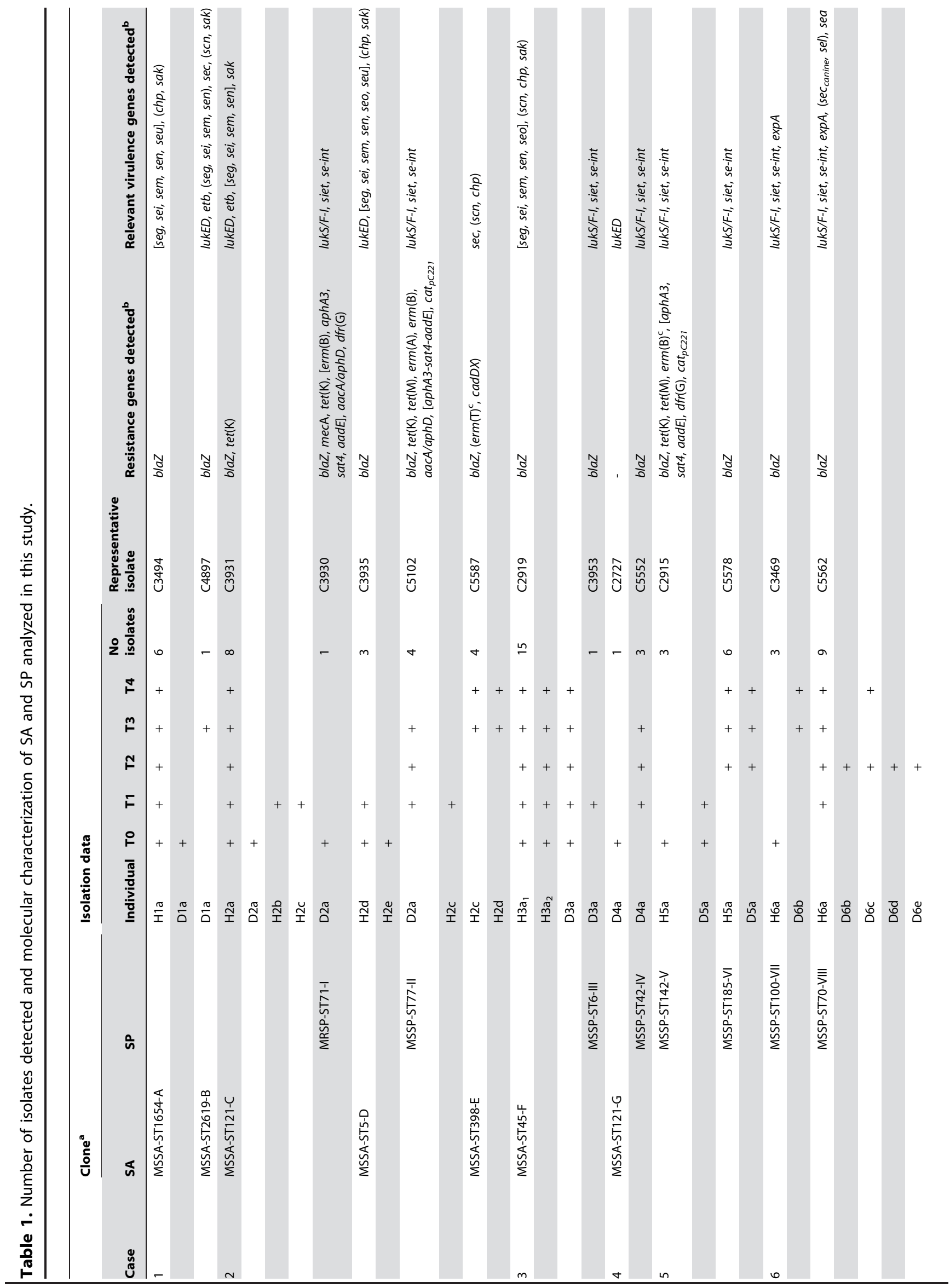




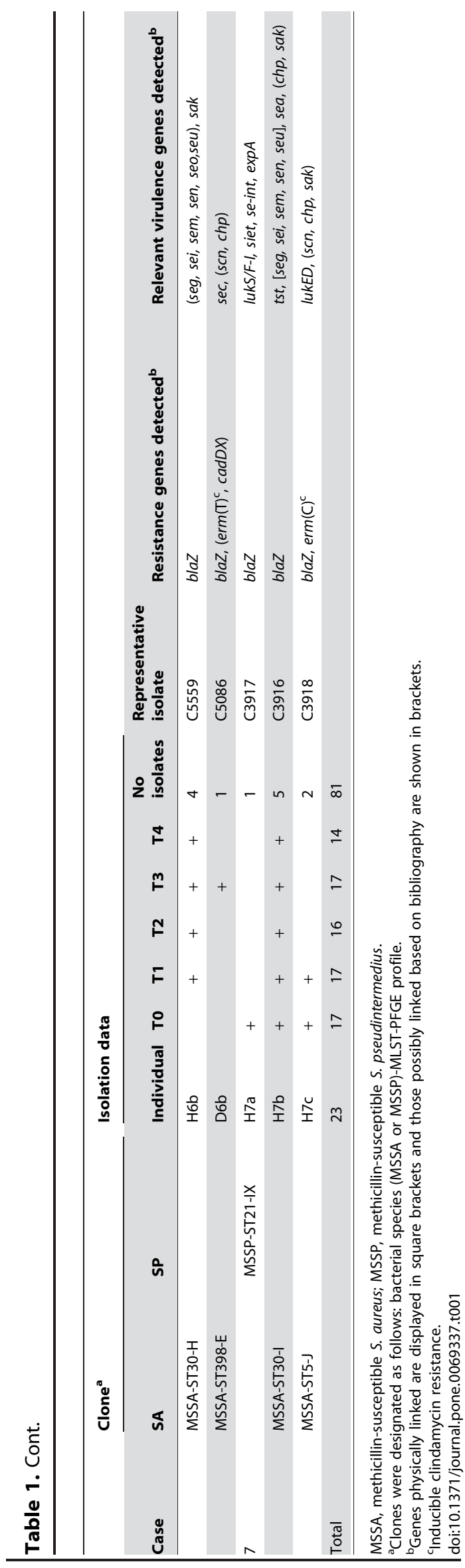

the SA clones were multidrug resistant (MDR) (resistance to at least three classes of antimicrobials). The majority of the SA clones (6/10) was susceptible to all antimicrobials or showed only penicillin resistance harboring the blaz gene. Two clones (MSSA-ST398-E, MSSA-ST5-J) presented additional MLS $_{\mathrm{B}}$ resistance $[\operatorname{erm}(\mathbf{C})$ or $\operatorname{erm}(\mathrm{T})]$, and a single clone (MSSA-ST121C) tetracycline resistance $[$ tet $(\mathrm{K})]$.

All SP isolates from the same clone presented identical antimicrobial resistance phenotype. A single sporadic MRSP clone (MRSP-ST71-I) and 2 MSSP (MSSP-ST77-II, MSSPST142-V) were MDR (Table 1). The remaining SP clones obtained were susceptible to all antimicrobials or showed only penicillin resistance (blaZ).

\section{Presence of Virulence Genes in SA and SP}

Virulence traits of SA and SP clones are shown in Table 1. All isolates lacked the PVL-toxin genes $l u k S / F-P V$. All SA representative strains (one isolate per clone, per household) but one carried enterotoxin genes, with $63.6 \%$ of them harboring different enterotoxin-gene-cluster combinations. Furthermore, $45.5 \%$ of them exhibited the leukocidin $l u k E / D$ genes and $18.2 \%$ of clones the exfoliatin gene etb. All SP representative isolates tested harbored the lukS/F-I, siet and se-int and $3(33.3 \%)$ the expA gene.

\section{SA and SP Species Distribution along the Sample Year}

Most positive owners (10/14) carried only SA throughout the sampling year. However, three owners only exhibited SP (Figure 2). Both, SA and SP were obtained along the study in only one person. In contrast, both bacterial species were recovered from a high number of positive dogs (4/9) along the study, with a single dog carrying only SA. Four animals were only positive for SP.

\section{SA and SP Carriage Status}

The carriage status of the investigated subjects over time is shown in Figure 3. Among the 16 owners, seven were persistent carriers of SA and 2 of SP. None of these individuals presented SA or SP co-carriage along the sampling period (Figure 1). Two humans were intermittent SA carriers. Two owners were sporadic SA carriers and 2 sporadic SP carriers. A single owner (H2b) was carrier of both bacterial species along the year (intermittent SA carrier and sporadic SP carrier) (Figure 1). Only 2 owners were non-carrier throughout the sample year.

The majority of dogs $(6 / 10)$ were sporadic nasal carriers of SP $(\mathrm{n}=3)$ or $\mathrm{SA}(\mathrm{n}=3)$. Three dogs were intermittent SP carriers and one dog was a SA intermittent carrier, with 2 of these animals being also sporadic SA carriers. Two animals were SP persistent carriers and one was a persistent carrier of SA. Among these, two dogs were additional sporadic SA and SP co-carriers (Figure 1). Remarkably, $40 \%$ of dogs belonged to 2 distinct carriage types depending on bacterial species. A single dog was non-carrier.

\section{Dynamics of the Interspecies Transmission Cases over Time}

Cases of direct SA transmission. All three households with presumed SA direct transmission (cases 1, 2 and 3) maintained the original SA clone in the owners implicated in the index cases while only one index dog (D3a) was a persistent carrier (MSSA-ST45-F) (Table 1, Figure 1).

In case 2 , index $\operatorname{dog} \mathrm{D} 2 \mathrm{a}$ revealed to be a persistent $\mathrm{SP}$ carrier due to the presence of a MDR SP clone (MSSP-ST77-II) in samplings T1, T2 and T3 (Table 1, Figure 1). A coexisting owner (H2b) harbored the aforementioned SP clone in sampling T1, what represents a novel sporadic case of direct SP interspecies 
Table 2. Different spa types present among the S. aureus clones per household during the sample period (T0-T4), positive individuals and correspondent clones.

\begin{tabular}{|c|c|c|c|c|c|}
\hline Case & spa & repeats & Clone & Sampling & Individual/s \\
\hline \multirow[t]{2}{*}{1} & t021 & $15-12-16-02-16-02-25-17-24$ & MSSA-ST1654-A & $\mathrm{T} 0, \mathrm{~T} 1, \mathrm{~T} 2, \mathrm{~T} 3, \mathrm{~T} 4$ & $\mathrm{H} 1 \mathrm{a}, \mathrm{D} 1 \mathrm{a}^{\mathrm{TO}}$ \\
\hline & t159 & $14-44-13-12-17-17-23-18-17$ & MSSA-ST2619-B & T3 & D1a \\
\hline \multirow[t]{3}{*}{2} & $\mathrm{t} 159$ & $14-44-13-12-17-17-23-18-17$ & MSSA-ST121-C & $\mathrm{T} 0, \mathrm{~T} 1, \mathrm{~T} 2, \mathrm{~T} 3, \mathrm{~T} 4$ & $\mathrm{H} 2 \mathrm{a}, \mathrm{H} 2 \mathrm{~b}^{T 1}, \mathrm{H} 2 \mathrm{c}^{\top 1}, \mathrm{D} 2 \mathrm{a}^{\mathrm{TO}}$ \\
\hline & t002 & $26-23-17-34-17-20-17-12-17-16$ & MSSA-ST5-D & T0, T1 & $\mathrm{H} 2 \mathrm{~d}, \mathrm{H} 2 \mathrm{e}^{\mathrm{TO}}$ \\
\hline & $\mathrm{t} 1451$ & $08-16-02-25-34-25$ & MSSA-ST398-E & $\mathrm{T} 3, \mathrm{~T} 4$ & $\mathrm{H} 2 \mathrm{~b}, \mathrm{H} 2 \mathrm{~d}$ \\
\hline \multirow[t]{3}{*}{3} & t073 & $08-16-02-16-13--17-34-16-34$ & MSSA-ST45-F & $\mathrm{T} 0, \mathrm{~T} 1, \mathrm{~T} 2, \mathrm{~T} 3, \mathrm{~T} 4$ & $\mathrm{H} 3 \mathrm{a}_{1}{ }^{\mathrm{TO}}{ }^{\mathrm{T} 3}, \mathrm{~T}^{4}, \mathrm{H} \mathrm{a}_{2}, \mathrm{D} 3 \mathrm{a}$ \\
\hline & $\mathrm{t} 116$ & $08-16-02-16-13-13-17-34-16-34$ & MSSA-ST45-F & $\mathrm{T} 1$ & $\mathrm{H} 3 \mathrm{a}_{1}$ \\
\hline & t026 & $08-16-----34$ & MSSA-ST45-F & $\mathrm{T} 2$ & $\mathrm{H} 3 \mathrm{a}_{1}$ \\
\hline 4 & $\mathrm{t} 151$ & $26-17-20-17-16$ & MSSA-ST121-G & T0 & D4a \\
\hline \multirow[t]{2}{*}{6} & $\mathrm{t} 1071$ & $26-23-12-23-02-34-34$ & MSSA-ST30-H & $\mathrm{T} 1, \mathrm{~T} 2, \mathrm{~T} 3, \mathrm{~T} 4$ & $\mathrm{H} 6 \mathrm{~b}$ \\
\hline & $\mathrm{t} 1451$ & $08-16-02-25-34-25$ & MSSA-ST398-E & T3 & D6b \\
\hline \multirow[t]{2}{*}{7} & t012 & $15-12-16-02-16-02-25-17-24-24$ & MSSA-ST30-I & $\mathrm{T} 0, \mathrm{~T} 1, \mathrm{~T} 2, \mathrm{~T} 3, \mathrm{~T} 4$ & $\mathrm{H} 7 \mathrm{~b}$ \\
\hline & t002 & $26-23-17-34-17-20-17-12-17-16$ & MSSA-ST5-J & $\mathrm{T} 0, \mathrm{~T} 1, \mathrm{~T} 2$ & $H 7_{c}$ \\
\hline
\end{tabular}

When an individual was not positive for the specific spa type in all indicated samplings the positive sampling is indicated in superscript. Boldface and dashes added to facilitate comparison of spa repeats in the different isolates of clone MSSA-ST45-F.

doi:10.1371/journal.pone.0069337.t002

transmission. It is interesting to underline the presence of a SA t1451 clone (MSSA-ST398-E) that belongs to the lineage ST398 in two owners (H2b and $\mathrm{H} 2 \mathrm{~d}$ ) in two consecutive samplings (T3$\mathrm{T} 4)$.

Indirect SA anthropozoonotic transmission. The dog from case 4 (D4a), positive for a MSSA-ST121-G isolate only in the initial sampling, was positive for a SP clone (MSSP-ST42-IV) in the three following samplings (Table 1, Figure 1), while its owner was a non SA or SP carrier.

Direct SP transmission. In case 5 , owner and $\operatorname{dog}(\mathrm{H} 5 \mathrm{a}$ and D5a) were persistent SP carriers. In the last 3 samplings (T2 to T4) a different SP clone (MSSP-ST185-V) from the index case was observed. (Table 1, Figure 1).

Gases of indirect SP zoonotic transmission. As for case 6 , the index human $(\mathrm{H} 6 \mathrm{a})$ was permanently colonized by SP, with MSSP-ST100-VII isolated in T0, and MSSP-ST70-VIII in the subsequent samplings (T1 to T4) (Table 1, Figure 1). Interestingly, both STs only differed in a silent mutation in allele tuf (tuf_2 at position T136A) and presented the exfoliatin gene expA (Table 1). The coexisting human (H6b) was a persistent carrier of a SA clone (MSSA-ST30-H). Remarkably, one dog (D6b) carried a MSSA t1451-ST398 isolate in one sampling (T3).

In case 7 , neither the index human $(\mathrm{H} 7 \mathrm{a})$ nor any of the coexisting individuals carried the original MSSP ST21 clone in subsequent samplings (Table 1, Figure 1).

\section{Co-carriage with MRCoNS and Molecular Characterization of Isolates}

Thirty-two MRCoNS isolates (28 S. epidermidis, 3 S. haemolyticus and 1 S. succinus) were detected along the four samplings from a high rate of owners (14/16) and in half of tested dogs (5/10), of which 19 (18 S. epidermidis and one S. haemolyticus) were present with SA or SP and further investigated (Figure 1). In addition to the $S$. haemolyticus clone (MRSH-6), 9 MRSE clones were observed by SmaI-PFGE, with clone 4 presenting two subclones $(4 \mathrm{a}-4 \mathrm{~b})$ (Table 3).

A total of $15.6 \%$ (10/64) of SA/SP positive samples also carried a MDR MRCoNS isolate. Half of MRCoNS clones were MDR carrying a wide variety of antimicrobial resistance genes (Table 3). Interestingly, several isolates of the same clones from cases 3 (MRSE-4) and 6 (MRSE-8) exhibited different antimicrobial resistance pheno- and/or genotypes to mupirocin over time (Table 3). Moreover, clone MRSE-9 in 2 individuals (H6b and D6c) presented different antimicrobial resistance profiles at the same sampling (T2). The trimethoprim resistance $d f r(\mathrm{G})$ gene was detected in clone MRSH-6 and MSSP-ST142-V in the same sampling (Table 3).

Half of SA or SP positive owners and 3 of the 9 positive dogs cocarried a MRCoNS isolate in at least one sampling. Among these, two owners $\left(\mathrm{H}_{3} \mathrm{a}_{1}\right.$ and $\left.\mathrm{H} 6 \mathrm{a}\right)$ were persistent carriers of both types of staphylococci (Figure 1, Table 3). In case 6, owner H6b and dog D6c harbored the identical MRSE-9 in sampling T2, representing a novel case of direct interspecies transmission, but this time for by MRCoNS isolates.

\section{Particular Traits of the SA ST398 Isolates}

Four SA t1451-ST398 isolates from two owners (H2b, H2d) at successive samplings (case 2) and one from an unrelated household (case 6) from a dog (D6b), belonged the same SA clone (MSSAST398-E) based on all molecular techniques performed (Table 1).



Figure 2. Distribution of investigated individuals (owners, dogs) related to the SA and/or SP carriage as a summary of the sampling year (TO-T4).

doi:10.1371/journal.pone.0069337.g002 


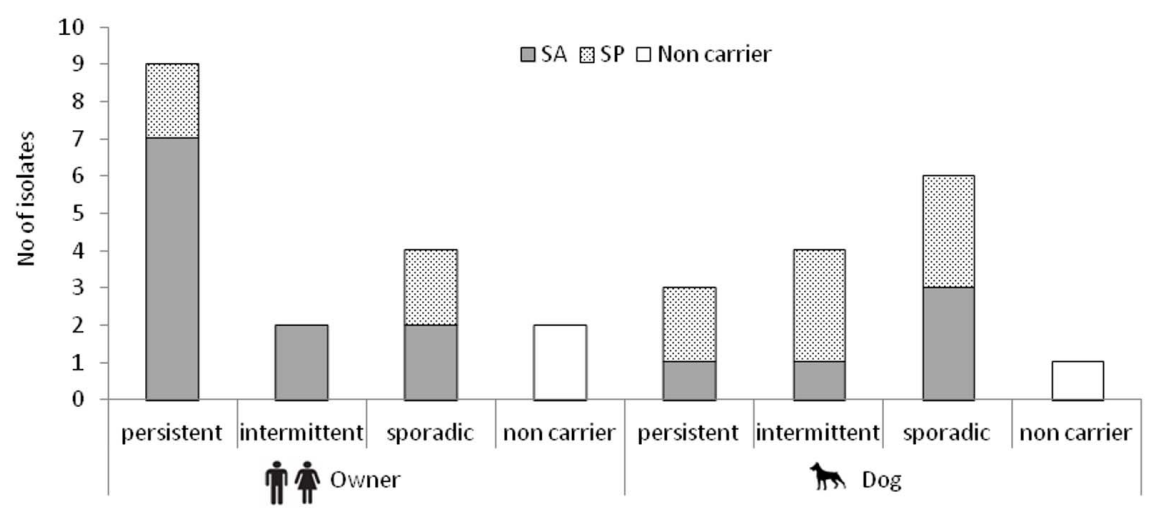

Figure 3. Number of investigated individuals (owners and dogs) with different S. aureus (SA) and S. pseudintermedius (SP) carriage status over the sample year (T0-T4). It should be noted that the sum is higher than the total number of isolates given that individuals could belong to 2 distinct carriage types depending on bacterial species.

doi:10.1371/journal.pone.0069337.g003

Only these isolates exhibited inducible $\mathrm{MLS}_{\mathrm{B}}$ resistance due to the presence of the $\operatorname{erm}(\mathrm{T})$ gene. PCR analysis for the presence of the cadmium resistance operon cadDX (recently described in physical linkage to $\operatorname{erm}(\mathrm{T})$ in a small MSSA ST398 plasmid, pUR3912 [43]) revealed their existence in the five isolates. All 5 isolates were tested for virulence determinants and evidenced the presence of the scn, $\operatorname{ch} p$ and the enterotoxin gene sec. Both positive owners live in an urban area with not contact to livestock or other farm animals. The positive dog lives in a rural area without direct contact to livestock.

\section{Discussion}

This is the first study on the dynamics of colonization of SA and SP in healthy pet-owning household members. All investigated households presented at least one individual positive for SA or SP in each of the samplings. In addition, the vast majority of investigated owners (14/16) and dogs (9/10) carried either SA or $\mathrm{SP}$ in at least one sampling, what evidences a real flow of both bacterial species within pet-owning household settings.

Based on the dynamics of colonization of SA it seems that the original source of SA was the human and that dogs serve as sporadic sources of human carriage, which may play a role in recolonization or maintenance. The household with an identical SA clone in owners and dog along the whole year (case 3) might be explained by the persistent carriage of both index persons. Interestingly, in this household, SA isolates (clone MSSA-ST45F) with three different $s p a$ types in serial samplings were recovered from one of the owners $\left(\mathrm{H}_{3} \mathrm{a}_{1}\right)$. The identical PFGE band profile in these $\mathrm{SA}$ isolates suggests evolution of its spa repeat composition rather than the presence of different clones. In this line, mutations over time within the same SA clone have been previously detected in SA longitudinal studies [45-48].

The fact that SP was maintained along the whole sample year in two owners reveals that humans can either be carriers for prolonged periods of time of this bacterial species or present easiness for recurrent acquisition. This observation suggests that the transmission capacities of SP, as well as its survival ability on non-natural hosts, may have been overlooked. With this respect, owner and dog from case 5 lived separately for several months between $\mathrm{T} 0$ and $\mathrm{T} 1$. The absence of $\mathrm{SP}$ in the owner in $\mathrm{T} 1$ suggests that the source of SP was the dog.

The presence of SP persistent owners, together with the absence of intermittent SP human carriers might point to favourable conditions in specific individuals to be colonized by SP. In contrast, both bacterial species were present in all carriage types in dogs, with SA being in most cases sporadically detected. This observation reflects the ability of dogs to be secondary reservoirs for staphylococcal propagation.

SA and SP carriage evolution showed different trends depending on the host from which they were recovered: (i) a single owner was positive for both bacterial species, what contrasts with the elevated number of positive dogs from which SA and SP was recovered (4/9), and (ii) SP was not detected in the SA-persistent owners, and SA was not present in the SP-persistent humans, while two of the three persistently colonized dogs were sporadic nasal carriers of the other coagulase positive staphylococci. These observations suggest that these bacteria might present a hostdependent behaviour and/or that they may interfere with each other's colonization in the human host.

While SA normally colonizes the nasal cavity of humans [1], SP seems to be more variably located in dogs, and perineum and mouth have been recently addressed as the body-sites with most $\mathrm{SP}$ recovery rates $[2,49]$. A recent study focused on the dynamics of SP in healthy dogs with multiple sampling sites, revealed that between 2 and 5 clones were present in the same animal, what is in agreement with our results. Replacement of SP clones in an individual microbiota over time has been previously reported as common in previous longitudinal studies in SP in dogs [50]. In contrast, the SA population structure was mostly maintained in the SA persistent individuals, what is in line with other longitudinal SA studies on humans [16]. To our knowledge, no information is available on preferential colonization sites of SA in dogs and SP in humans, what warrants further investigations.

MSSA strains belonging to the same genetic background as pandemic MRSA clones (CG45, CG121, CC30, CG398, CG5) were collected from humans, of which CG121 and CG398 were also sporadically detected in dogs. The elevated virulence gene content of all SA clones detected enhances the importance to control possible sources for acquisition of potential pathogenic bacteria. In addition, all SA clones but one, detected in a dog (MSSA-ST121-G), harbored genes implicated in evasion of the human immune system, what responds to a human adaptation.

Interestingly, all 5 MSSA-ST398-E isolates (4 from 2 humans, 1 from dog) carried the $\mathrm{MLS}_{\mathrm{B}}$ resistance gene $\operatorname{erm}(\mathrm{T})$ and the cadmium resistance operon $\operatorname{cadDX}$, which has been recently described to be co-located within pUR3912 [44]. The ability of this small plasmid to integrate within the chromosomal DNA [51] 
Table 3. Characteristics of the MRCoNS recovered in the SA/SP positive individuals per household.

\begin{tabular}{|c|c|c|c|c|c|c|c|c|}
\hline \multirow[b]{2}{*}{ Case } & \multirow[b]{2}{*}{ Clone } & \multirow[b]{2}{*}{ Individual } & \multirow[b]{2}{*}{ ISampling } & \multirow[b]{2}{*}{ Isolate } & \multirow{2}{*}{$\begin{array}{l}\text { Phenotype of } \\
\text { resistance }^{a}\end{array}$} & \multirow{2}{*}{$\begin{array}{l}\text { Antimicrobial resistance profile } \\
\text { Resistance genes detected }\end{array}$} & \multicolumn{2}{|c|}{ Concomitant SA/SP clone } \\
\hline & & & & & & & SA & SP \\
\hline 1 & MRSE-1 & D1a & T3 & C4812 & P-O-F-E & blaZ, mecA, mphC, msrA/msrB & MSSA-ST2619-B & \\
\hline \multirow[t]{2}{*}{2} & MRSE-2 & $\mathrm{H} 2 \mathrm{a}$ & $\mathrm{T} 2$ & C4807 & P-O-F-E-M & blaZ, mecA, mphC, msrA/msrB, mupA & MSSA-ST121-C & \\
\hline & MRSE-3 & $\mathrm{H} 2 \mathrm{~d}$ & T4 & C5588 & P-O-F & blaZ, mecA & MSSA-ST398-E & \\
\hline \multirow[t]{6}{*}{3} & MRSE-4a & $\mathrm{H} 3 \mathrm{a}_{1}$ & $\mathrm{~T} 1$ & C3956 & P-O-F & blaz, mecA & MSSA-ST45-F & \\
\hline & MRSE-4b & $\mathrm{H}_{3} \mathrm{a}_{1}$ & T3 & C5097 & P-O-F-M & blaZ, mесA, mupA & MSSA-ST45-F & \\
\hline & MRSE-4b & $\mathrm{H}^{3} \mathrm{a}_{2}$ & $\mathrm{~T} 2$ & C4798 & P-O-F-M & blaZ, mecA, mupA & MSSA-ST45-F & \\
\hline & MRSE-5 & $\mathrm{H}_{3} \mathrm{a}_{2}$ & $\mathrm{~T} 1$ & C3958 & P-O-F-T-E-To & blaZ, mecA, tet $(\mathrm{K}), \operatorname{tet}(\mathrm{M}), m p h C, m s r A / m s r B, a a d D$ & MSSA-ST45-F & \\
\hline & MRSE-5 & $\mathrm{H} 3 \mathrm{a}_{1}$ & $\mathrm{~T} 2$ & C4796 & P-O-F-T-E-To & blaZ, mecA, tet(K), tet(M), mphC, msrA/msrB, aadD & MSSA-ST45-F & \\
\hline & MRSE-5 & $\mathrm{H}_{3} \mathrm{a}_{1}$ & T4 & C5573 & P-O-F-T-E-To & blaZ, mecA, tet $(\mathrm{K}), \operatorname{tet}(\mathrm{M}), m p h C, m s r A / m s r B, a a d D$ & MSSA-ST45-F & \\
\hline \multirow[t]{2}{*}{5} & MRSH-6 & D5a & $\mathrm{T} 1$ & C3960 & P-O-F-E-X-Fu ${ }^{b}$ & blaZ, mecA, mphC, msrA/msrB, dfr(A), dfr(G) & & MSSP-ST142-V \\
\hline & MRSE-7 & D5a & T4 & C5577 & P-O-F & blaz, mecA & & MSSP-ST185-VI \\
\hline \multirow[t]{7}{*}{6} & MRSE-8 & H6a & $\mathrm{T} 1$ & C3945 & P-O-F-G-K-M-Fu ${ }^{b}$ & blaZ, mecA, aacA/aphD, mupA & & MSSP-ST70-VIIIa \\
\hline & MRSE-8 & H6a & $\mathrm{T} 2$ & C4791 & P-O-F-G-K-M-Fu ${ }^{b}$ & blaZ, mecA, aacA/aphD, mupA & & MSSP-ST70-VIIIlb \\
\hline & MRSE-8 & H6a & T3 & C5093 & P-O-F-G-K-M-Fu ${ }^{\mathrm{b}}$ & blaZ, mecA, aacA/aphD, mupA & & MSSP-ST70-VIIIlb \\
\hline & MRSE-8 & H6a & $\mathrm{T} 4$ & C5564 ${ }^{\mathrm{C}}$ & P-O-F-G-K-Fu ${ }^{b}$ & blaZ, mecA, aacA/aphD, mupA & & MSSP-ST70-VIIIC \\
\hline & MRSE-8 & $\mathrm{H} 6 \mathrm{~b}$ & T4 & C5558 & P-O-F-G-K-M-Fu ${ }^{\mathrm{b}}$ & blaZ, mecA, aacA/aphD, mupA & & MSSP-ST70-VIIIC \\
\hline & MRSE-9 & H6b & $\mathrm{T} 2$ & C4789 & P-O-F-G-To-K & mecA, aacA/aphD & MSSA-ST30-H & \\
\hline & MRSE-9 & D6c & $\mathrm{T} 2$ & C4787 & P-O-F-T & blaZ, mecA, tet $(\mathrm{K}), \operatorname{tet}(\mathrm{M})$ & & MSSP-ST70-VIIIlb \\
\hline 7 & MRSE-10 & $\mathrm{H} 7 \mathrm{~b}$ & T4 & C5581 & P-O-F-E & blaZ, mecA, mphC, msrA/msrB & MSSA-ST30-J & \\
\hline
\end{tabular}

MRSE, methicillin-resistant S. epidermidis; MRSH, methicillin-resistant S. haemolyticus.

${ }^{\mathrm{a} P}$, penicillin; O, oxacillin; F, cefoxitin; E, erythromycin; M, mupirocin; $\mathrm{Cp}$, ciprofloxacin; To, tobramycin; X, co-trimoxazol; Fu, fusidic acid; G, gentamicin; K, kanamycin; T, tetracycline.

${ }^{\mathrm{b}}$ Fusidic acid resistance genes fusB and fus $C$ were not detected.

isolate $C 5564$ was mupirocin susceptible regardless the presence of the mupA gene.

doi:10.1371/journal.pone.0069337.t003

suggests a plausible clonal spread of pUR3912 among animalindependent MSSA ST398 isolates. Positive owners did not have direct contact to rural areas or farm animals and the presence of the IEC genes points to a human adaptation [52]. The detection of an enterotoxin gene $(\mathrm{sec})$ in all 5 isolates is remarkable, given that these toxin genes are normally absent among MRSA ST398 isolates. The presence of the same ApaI-PFGE band profile between unrelated individuals suggests a clonal distribution.

Co-carriage of SA and MRCoNS has been previously reported to have a negative association due to competition for the same ecological niche [53,54]. Moreover, S. epidermidis is considered to "protect" the host from SA colonization [54]. Half of SA or SP positive owners also presented MRCoNS in at least one sampling, what reflects a conceivable punctual coexistence of such bacteria. Further, the persistent co-carriage of MRSE and SA or SP observed in two owners $(12.5 \%$ of investigated humans) may represent a source of antimicrobial resistance genes for SA and SP.

The detection of a MRSE sporadic case of interspecies transmission (which direction remains unknown) that involved isolates with different antimicrobial resistance profiles evidences transmissibility potential. The presence of mupirocin resistance mирA gene in $42 \%(8 / 19)$ of MRCoNS isolates represents a potential risk for acquisition during SA/SP intended decolonization with mupirocin-based treatments. Further, the differential evolution of mupirocin resistance in some isolates of the same clone deserves continued surveillance. Although the acceptance of exogenous genetic material between staphylococci does seem to require special circumstances not yet properly understood [31], transmission of the mecA gene between MRCoNS and SA has been previously assumed [28,29,31].

\section{Conclusions}

This study gains knowledge in the ecology of SA, SP and MRCoNS in humans and in-contact dogs and represents a first approach to understand the role that dogs may play in the epidemiology of SA and SP in the human host over time. The apparent adaptability of SP to colonize dog owners who do not carry SA, as well as great predisposition of these animals to be SA carriers should be taken into account in subsequent developments of infection control measurements. Moreover, the human-associated MSSA ST398 is shown to be transferred and maintained within household members. Further longitudinal studies on SA and SP with a larger cohort in humans and in-contact dogs are essential for confident assertions of the implication of pet-owning as increased risk factor to acquire and maintain potentially pathogenic bacteria.

\section{Acknowledgments}

The authors wish to thank all household members for their excellent cooperation and Javier Ferrer-Frías for his collaboration on sample collection.

Part of these data was presented at the $3^{\text {rd }}$ ASM Conference on Antimicrobial resistance in zoonotic bacteria and foodborne pathogens in animals, humans and the environment. Aix-en-Provence, France, 26-29 June 2012 . 


\section{Author Contributions}

Conceived and designed the experiments: EGS MZ CT. Performed the experiments: EGS CL SG. Analyzed the data: EGS CT SG CL MZ.

\section{References}

1. Wertheim HF, Melles DC, Vos MC, van Leeuwen W, van Belkum A, et al. (2005) The role of nasal carriage in Staphylococcus aureus infections. Lancet Infect Dis 5: 751-762.

2. Bannoehr J, Guardabassi L (2012) Staphylococcus pseudintermedius in the dog: taxonomy, diagnostics, ecology, epidemiology and pathogenicity. Vet Dermatol 23: 253-266.

3. Hanselman BA, Kruth SA, Rousseau J, Weese JS (2009) Coagulase positive staphylococcal colonization of humans and their household pets. Can Vet J 50: 954-958.

4. Boost MV, O'Donoghue MM, James A (2008) Prevalence of Staphylococcus aureus carriage among dogs and their owners. Epidemiol Infect 136: 953-964.

5. Weese JS (2010) Methicillin-resistant Staphylococcus aureus in animals. ILAR J 51: 233-244.

6. Weese JS, Dick H, Willey BM, McGeer A, Kreiswirth BN, et al. (2006) Suspected transmission of methicillin-resistant Staphylococcus aureus between domestic pets and humans in veterinary clinics and in the household. Vet Microbiol 115: 148-155.

7. Gómez-Sanz E, Torres C, Lozano C, Zarazaga M (2013) High diversity of Staphylococcus aureus and Staphylococcus pseudintermedius lineages and toxigenic traits in healthy pet-owning household members. Underestimating normal household contact? Comp Immunol Microbiol Infect Dis 36: 83-94.

8. Mahoudeau I, Delabranche X, Prevost G, Monteil H, Piemont Y (1997) Frequency of isolation of Staphylococcus intermedius from humans. J Clin Microbiol 35: 2153-2154.

9. Talan DA, Staatz D, Staatz A, Overturf GD (1989) Frequency of Staphylococcus intermedius as human nasopharyngeal flora. J Clin Microbiol 27: 2393.

10. Walther B, Hermes J, Cuny C, Wieler LH, Vincze S, et al. (2012) Sharing more than friendship-nasal colonization with coagulase-positive staphylococci (CPS) and co-habitation aspects of dogs and their owners. PLoS One 7: e35197.

11. Bond R, Loeffler A (2012) What's happened to Staphylococcus intermedius? Taxonomic revision and emergence of multi-drug resistance. J Small Anim Pract 53: 147-154

12. Harvey RG, Marpless RR, Noble WC (1994) Nasal Carriage of Staphylococcus intermedius in Humans in Contact with Dogs. Microb Ecol Health D 7: 225-227.

13. van Duijkeren E, Kamphuis M, van der Mije IC, Laarhoven LM, Duim B, et al. (2011) Transmission of methicillin-resistant Staphylococcus pseudintermedius between infected dogs and cats and contact pets, humans and the environment in households and veterinary clinics. Vet Microbiol 150: 338-343.

14. Laarhoven LM, de Heus P, van Luijn J, Duim B, Wagenaar JA, et al. (2011) Longitudinal study on methicillin-resistant Staphylococcus pseudintermedius in households. PLoS One 6: e27788.

15. Van Belkum A, Riewarts Eriksen NH, Sijmons M, Van Leeuwen W, Van den Bergh M, et al. (1997) Coagulase and protein A polymorphisms do not contribute to persistence of nasal colonisation by Staphylococcus aureus. J Med Microbiol 46: 222-232.

16. VandenBergh MF, Yzerman EP, van Belkum A, Boelens HA, Sijmons M, et al. (1999) Follow-up of Staphylococcus aureus nasal carriage after 8 years: redefining the persistent carrier state. J Clin Microbiol 37: 3133-3140.

17. Lomholt H, Andersen KE, Kilian M (2005) Staphylococcus aureus clonal dynamics and virulence factors in children with atopic dermatitis. J Invest Dermatol 125: 977-982.

18. Pan ES, Diep BA, Charlebois ED, Auerswald C, Carleton HA, et al. (2005) Population dynamics of nasal strains of methicillin-resistant Staphylococcus aureusand their relation to community-associated disease activity. J Infect Dis 192: 811-818.

19. Lebon A, Labout JA, Verbrugh HA, Jaddoe VW, Hofman A, et al. (2008) Dynamics and determinants of Staphylococcus aureus carriage in infancy: the Generation R Study. J Clin Microbiol 46: 3517-21.

20. VasanthaKumari N, Alshrari AS, Rad EG, Moghaddam HG, van Belkum A, et al. (2009) Highly dynamic transient colonization by Staphylococcus aureus in healthy Malaysian students. J Med Microbiol 58: 1531-1532.

21. Sakwinska O, Blanc DS, Lazor-Blanchet C, Moreillon M, Giddey M, et al. (2010) Ecological temporal stability of Staphylococcus aureus nasal carriage. J Clin Microbiol 48: 2724-2728.

22. Lautenbach E, Tolomeo P, Nachamkin I, Hu B, Zaoutis TE (2010) The impact of household transmission on duration of outpatient colonization with methicillin-resistant Staphylococcus aureus. Epidemiol Infect 138: 683-685.

23. Graveland H, Wagenaar JA, Bergs K, Heesterbeek H, Heederik D (2011) Persistence of livestock associated MRSA CC398 in humans is dependent on intensity of animal contact. PLoS One 6: e16830.

24. Lozano C, Aspiroz C, Lasarte JJ, Gómez-Sanz E, Zarazaga M, et al. (2011) Dynamic of nasal colonization by methicillin-resistant Staphylococcus aureus ST398 and ST1 after mupirocin treatment in a family in close contact with pigs. Comp Immunol Microbiol Infect Dis 34: e1-7.

25. Diekema DJ, Pfaller MA, Schmitz FJ, Smayevsky J, Bell J, et al. (2001) Survey of infections due to Staphylococcus species: frequency of occurrence and antimicrobial
Contributed reagents/materials/analysis tools: EGS CT SC CL MZ. Wrote the paper: EGS CT MZ.

susceptibility of isolates collected in the United States, Canada, Latin America, Europe, and the Western Pacific region for the SENTRY Antimicrobial Surveillance Program, 1997-1999. Clin Infect Dis 32: S114-132.

26. John JF, Harvin AM (2007) History and evolution of antibiotic resistance in coagulase-negative staphylococci: Susceptibility profiles of new anti-staphylococcal agents. Ther Clin Risk Manag 3: 1143-1152.

27. Lebeaux D, Barbier F, Angebault C, Benmahdi L, Ruppé E, et al. (2012) Evolution of nasal carriage of methicillin-resistant coagulase-negative staphylococci in a remote population. Antimicrob Agents Chemother 56: 315-323.

28. Hanssen AM, Kjeldsen G, Sollid JU (2004) Local variants of Staphylococcal cassette chromosome mec in sporadic methicillin-resistant Staphylococcus aureus and methicillin-resistant coagulase-negative Staphylococci: evidence of horizontal gene transfer? Antimicrob Agents Chemother 48: 285-296.

29. Berglund C, Söderquist B (2008) The origin of a methicillin-resistant Staphylococcus aureus isolate at a neonatal ward in Sweden-possible horizontal transfer of a staphylococcal cassette chromosome mec between methicillinresistant Staphylococcus haemolyticus and Staphylococcus aureus. Clin Microbiol Infect 14: 1048-1056.

30. Barbier F, Ruppé E, Hernandez D, Lebeaux D, Francois P, et al. (2010) Methicillin-resistant coagulase-negative staphylococci in the community: high homology of SCCmec IVa between Staphylococcus epidermidis and major clones of methicillin-resistant Staphylococcus aureus. J Infect Dis 202: 270-281.

31. Bloemendaal AL, Brouwer EC, Fluit AC (2010) Methicillin resistance transfer from Staphylocccus epidermidis to methicillin-susceptible Staphylococcus aureus in a patient during antibiotic therapy. PLoS One 5: e11841.

32. Baron F, Cochet MF, Pellerin JL, Ben Zakour N, Lebon A, et al. (2004) Development of a PCR test to differentiate between Staphylococcus aureus and Staphylococcus intermedius. J Food Prot 67: 2302-2305.

33. Bannoehr J, Franco A, Iurescia M, Battisti A, Fitzgerald JR (2009) Molecular diagnostic identification of Staphylococcus pseudintermedius. J Clin Microbiol 47: $469-471$.

34. Gómez-Sanz E, Torres C, Lozano C, Fernández-Pérez R, Aspiroz C, et al. (2010) Detection, molecular characterization, and clonal diversity of methicillinresistant Staphylococcus aureus CC398 and CG97 in Spanish slaughter pigs of different age groups. Foodborne Pathog Dis 7: 1269-1277.

35. Poyart C, Quesne G, Boumaila C, Trieu-Cuot P (2001) Rapid and accurate species-level identification of coagulase-negative staphylococci by using the sodA gene as a target. J Clin Microbiol 39: 4296-4301.

36. Murchan S, Kaufmann ME, Deplano A, de Ryck R, Struelens M, et al. (2003) Harmonization of pulsed-field gel electrophoresis protocols for epidemiological typing of strains of methicillin-resistant Staphylococcus aureus: a single approach developed by consensus in 10 European laboratories and its application for tracing the spread of related strains. J Clin Microbiol 41: 1574-1585.

37. Kadlec K, Ehricht R, Monecke S, Steinacker U, Kaspar H, et al. (2009) Diversity of antimicrobial resistance pheno- and genotypes of methicillinresistant Staphylococcus aureus ST398 from diseased swine. J Antimicrob Chemother 64: 1156-1164.

38. Perreten V, Kadlec K, Schwarz S, Grönlund Andersson U, Finn M, et al. (2010) Clonal spread of methicillin-resistant Staphylococcus pseudintermedius in Europe and North America: an international multicentre study. J Antimicrob Chemother 65: 1145-1154.

39. Tenover FC, Arbeit RD, Goering RV, Mickelsen PA, Murray BE, et al. (1995) Interpreting chromosomal DNA restriction patterns produced by pulsed-field gel electrophoresis: criteria for bacterial strain typing. J Clin Microbiol 33: 22332239.

40. Harmsen D, Claus H, Witte W, Rothgänger J, Claus H, et al. (2003) Typing of methicillin-resistant Staphylococcus aureus in a university hospital setting by using novel software for spa repeat determination and database management. J Clin Microbiol 41: 5442-5448.

41. Clinical and Laboratory Standards Institute (2012) Performance standards for antimicrobial susceptibility testing; Eighteenth informational supplement. CLSI Document M100-S22 Wayne PA.

42. Gómez-Sanz E, Torres C, Lozano C, Sáenz Y, Zarazaga M (2011) Detection and characterization of methicillin-resistant Staphylococcus pseudintermedius in healthy dogs in La Rioja, Spain. Comp Immunol Microbiol Infect Dis 34: 447-453.

43. van Wamel WJ, Rooijakkers SH, Ruyken M, van Kessel KP, van Strijp JA (2006) The innate immune modulators staphylococcal complement inhibitor and chemotaxis inhibitory protein of Staphylococcus aureus are located on betahemolysin-converting bacteriophages. J Bacteriol 188: 1310-1315.

44. Gómez-Sanz E, Kadlec K, Feßler AT, Billerbeck C, Zarazaga M, et al. (2013) Analysis of a novel erm( $\mathrm{T})$ - and $c a d D X$-carrying plasmid from methicillinsusceptible Staphylococcus aureus ST398-t571 of human origin. J Antimicrob Chemother 68: 471-473.

45. van Belkum A, Riewerts Eriksen N, Sijmons M, van Leeuwen W, VandenBergh M, et al. (1996) Are variable repeats in the spa gene suitable targets for 
epidemiological studies of methicillin-resistant Staphylococcus aureus strains? Eur J Clin Microbiol Infect Dis 15: 768-770.

46. Kahl BC, Mellmann A, Deiwick S, Peters G, Harmsen D (2005) Variation of the polymorphic region $\mathrm{X}$ of the protein A gene during persistent airway infection of cystic fibrosis patients reflects two independent mechanisms of genetic change in Staphylococcus aureus. J Clin Microbiol 43: 502-505.

47. Kuhn G, Francioli P, Blanc DS (2007) Double-locus sequence typing using $c l f B$ and spa, a fast and simple method for epidemiological typing of methicillinresistant Staphylococcus aureus. J Clin Microbiol 45: 54-62.

48. Sakwinska O, Blanc DS, Lazor-Blanchet C, Moreillon M, Giddey M, et al. (2010) Ecological temporal stability of Staphylococcus aureus nasal carriage. J Clin Microbiol 48: 2724-2728.

49. Paul NC, Bärgman SC, Moodley A, Nielsen SS, Guardabassi L (2012) Staphylococcus pseudintermedius colonization patterns and strain diversity in healthy dogs: a cross-sectional and longitudinal study. Vet Microbiol 160: 420-427.
50. Hartmann FA, White DG, West SE, Walker RD, Deboer DJ (2012) Molecular characterization of Staphylococcus intermedius carriage by healthy dogs and comparison of antimicrobial susceptibility patterns to isolates from dogs with pyoderma. Vet Microbiol 108: 119-131

51. Gómez-Sanz E, Zarazaga M, Kadlec K, Schwarz S, Torres C (2013) Chromosomal integration of the novel methicillin-susceptible Staphylococcus aureus ST398 plasmid pUR3912 of human origin. Clin Microbiol Infect in press.

52. McCarthy AJ, van Wamel W, Vandendriessche S, Larsen J, Denis O, et al. (2012) Staphylococcus aureus CC398 Clade Associated with Human-to-Human Transmission. Appl Environ Microbiol 78: 8845-8848.

53. Weidenmaier C, Goerke C, Wolz C (2012) Staphylococcus aureus determinants for nasal colonization. Trends Microbiol 20: 243-250.

54. Johannessen M, Sollid JE, Hanssen AM (2012) Host- and microbe determinants that may influence the success of $S$. aureus colonization. Front Cell Infect Microbiol 2: 56 